Bull. Korean Math. Soc. 50 (2013), No. 2, pp. 417-430

http://dx.doi.org/10.4134/BKMS.2013.50.2.417

\title{
CHARACTERIZATIONS OF GEOMETRICAL PROPERTIES OF BANACH SPACES USING $\psi$-DIRECT SUMS
}

\author{
Zhinua Zhang, Lan Shu, Jun Zheng, and Yuling Yang
}

\begin{abstract}
Let $X$ be a Banach space and $\psi$ a continuous convex function on $\Delta_{K+1}$ satisfying certain conditions. Let $(X \oplus X \oplus \cdots \oplus X)_{\psi}$ be the $\psi$-direct sum of $X$. In this paper, we characterize the $K$ strict convexity, $K$ uniform convexity and uniform non- $l_{1}^{N}$-ness of Banach spaces using $\psi$-direct sums.
\end{abstract}

\section{Introduction}

A norm $\|\cdot\|$ on $\mathbb{C}^{n}$ is said to be absolute if

$$
\left\|\left(x_{1}, x_{2}, \ldots, x_{n}\right)\right\|=\left\|\left(\left|x_{1}\right|,\left|x_{2}\right|, \ldots,\left|x_{n}\right|\right)\right\| \quad \text { for any }\left(x_{1}, x_{2}, \ldots, x_{n}\right) \in \mathbb{C}^{n}
$$

and normalized if

$$
\|(1,0, \ldots, 0)\|=\|(0,1,0, \ldots, 0)\|=\cdots=\|(0, \ldots, 0,1)\| .
$$

The $l_{p}$-norms are such examples:

$$
\left\|\left(x_{1}, x_{2}, \ldots x_{n}\right)\right\|_{p}=\left\{\begin{array}{l}
\left(\left|x_{1}\right|^{p}+\left|x_{2}\right|^{p}+\cdots+\left|x_{n}\right|^{p}\right)^{\frac{1}{p}}, \quad 1 \leq p<\infty \\
\max \left\{\left|x_{1}\right|,\left|x_{2}\right|, \ldots,\left|x_{n}\right|\right\}, \quad p=\infty .
\end{array}\right.
$$

Let $A N_{n}$ be the family of all absolute normalized norms on $\mathbb{C}^{n}$. When $n=$ 2 Bonsall and Duncan [2] showed the following characterization of absolute normalized norms on $\mathbb{C}^{2}$. Namely, the set $A N_{2}$ of all absolute normalized norms on $\mathbb{C}^{2}$ is in one-to-one correspondence with the set $\Psi_{2}$ of all continuous convex functions on $[0,1]$ satisfying $\psi(0)=\psi(1)=1$ and $\max \{1-t, t\} \leq \psi(t) \leq$ $1,0 \leq t \leq 1$. The correspondence is given by

$$
\psi(t)=\|(1-t, t)\|, \quad 0 \leq t \leq 1 .
$$

Received September 8, 2011; Revised December 8, 2011.

2010 Mathematics Subject Classification. 46B25, 46b20, 46B99.

Key words and phrases. absolute norm, $K$ strict convexity, $K$ uniform convexity, uniform non- $l_{1}^{N}$-ness.

The first and second authors are supported by Nation Natural Science Foundation of China(No. 11071178). 
Indeed, for any $\psi \in \Psi_{2}$, define

$$
\|(z, w)\|_{\psi}= \begin{cases}(|z|+|w|) \psi\left(\frac{|w|}{|z|+|w|}\right), & (z, w) \neq(0,0) \\ 0, & (z, w)=(0,0)\end{cases}
$$

By calculation we have $\|\cdot\|_{\psi} \in A N_{2}$ and $\|\cdot\|_{\psi}$ satisfies (1). From this result, there are plenty of concrete absolute normalized norms of $\mathbb{C}^{2}$ which are not $l_{p}$-type.

In [13] K.-S. Saito, M. Kato and Y. Takahashi generalized the result to $\mathbb{C}^{n}$. Before stating it, we give some notations. For each $n \in N$ with $n \geq 2$, we put

$$
\Delta_{n}=\left\{\left(t_{1}, t_{2}, t_{3}, \ldots, t_{n-1}\right) \in \mathbb{R}^{n-1}: t_{j} \geq 0, \sum_{j=1}^{n-1} t_{j} \leq 1\right\}
$$

and define the set $\Psi_{n}$ of all continuous convex functions on $\Delta_{n}$ satisfying the following conditions:

$$
\psi(0,0, \ldots, 0)=\psi(1,0, \ldots, 0)=\cdots=\psi(0, \ldots, 0,1)
$$

$\psi\left(t_{1}, t_{2}, \ldots, t_{n-1}\right) \geq\left(t_{1}+t_{2}+\cdots+t_{n-1}\right) \psi\left(\frac{t_{1}}{\sum_{i=1}^{n-1} t_{i}}, \ldots, \frac{t_{n-1}}{\sum_{i=1}^{n-1} t_{i}}\right)$, if $\sum_{i=1}^{n-1} t_{i} \neq 0$,

$\left(A_{2}\right) \quad \psi\left(t_{1}, t_{2}, \ldots, t_{n-1}\right) \geq\left(1-t_{1}\right) \psi\left(0, \frac{t_{2}}{1-t_{1}}, \ldots, \frac{t_{n-1}}{1-t_{1}}\right)$, if $t_{1} \neq 1$

$\left(A_{3}\right) \quad \psi\left(t_{1}, t_{2}, \ldots, t_{n-1}\right) \geq\left(1-t_{2}\right) \psi\left(\frac{t_{1}}{1-t_{2}}, 0, \ldots, \frac{t_{n-1}}{1-t_{2}}\right)$, if $t_{2} \neq 1$

$\left(A_{n}\right)$

$$
\psi\left(t_{1}, t_{2}, \ldots, t_{n-1}\right) \geq\left(1-t_{n-1}\right) \psi\left(\frac{t_{1}}{1-t_{n-1}}, \ldots, \frac{t_{n-2}}{1-t_{n-1}}, 0\right), \text { if } t_{n-1} \neq 1 .
$$

K.-S. Saito, M. Kato and Y. Takahashi in [13] showed that, for each $n \in \mathbb{N}$ with $n \geq 2, A N_{n}$ and $\Psi_{n}$ are in one-to-one correspondence under the following equation:

(2) $\psi\left(t_{1}, \ldots, t_{n-1}\right)=\left\|\left(1-\sum_{j=1}^{n-1} t_{j}, t_{1}, \ldots, t_{n-1}\right)\right\|,\left(t_{1}, \ldots, t_{n-1}\right) \in \Delta_{n}$. 
Indeed, for any $\psi \in \Psi_{n}$, the norm $\|\cdot\|_{\psi}$ on $\mathbb{C}^{n}$ is defined as

$$
\left\|\left(x_{0}, x_{1}, \ldots, x_{n-1}\right)\right\|_{\psi}=\left\{\begin{array}{c}
\left(\sum_{i=0}^{n-1}\left|x_{i}\right|\right) \psi\left(\frac{\left|x_{1}\right|}{\sum_{i=0}^{n-1}\left|x_{i}\right|}, \ldots, \frac{\left|x_{n-1}\right|}{\sum_{i=0}^{n-1}\left|x_{i}\right|}\right) \\
\left(x_{0}, x_{1}, \ldots, x_{n-1}\right) \neq(0, \ldots, 0) \\
0, \quad\left(x_{0}, x_{1}, \ldots, x_{n-1}\right)=(0, \ldots, 0) .
\end{array}\right.
$$

Moreover, M. Kato, K.-S. Saito and Tamura in [6] introduced the $\psi$-direct sums $\left(X_{1} \oplus X_{2} \bigoplus \cdots \oplus X_{n}\right)_{\psi}$ as follows. Let $X_{1}, X_{2}, \ldots, X_{n}$ be Banach spaces and let $\psi \in \Psi_{n}$. Then the product space $X_{1} \times X_{2} \times \cdots \times X_{n}$ with the norm

$$
\left\|\left(x_{1}, x_{2}, \ldots, x_{n}\right)\right\|_{\psi}=\left\|\left(\left\|x_{1}\right\|,\left\|x_{2}\right\|, \ldots,\left\|x_{n}\right\|\right)\right\|_{\psi}, \quad x_{i} \in X_{i}, 1 \leq i \leq n,
$$

is a Banach space which is denoted by $\left(X_{1} \oplus X_{2} \bigoplus \cdots \oplus X_{n}\right)_{\psi}$. They showed that $\left(X_{1} \oplus X_{2} \oplus \cdots \oplus X_{n}\right)_{\psi}$ is strictly convex (uniformly convex) if and only if $X_{1}, X_{2}, \ldots, X_{n}$ is strictly convex (uniformly convex) and $\psi \in \Psi_{n}$ is strictly convex. In [7] the authors presented that $X \bigoplus_{\psi} Y$ is uniformly non-square if and only if $X$ and $Y$ are uniformly non-square and $\psi \neq \psi_{1}, \psi_{\infty}$. Since the introduction of $\psi$-direct sums of Banach spaces, it has attracted plenty of attention and been treated by several authors (cf. [3, 4, 5, 12, 16]).

In particular, K.-I. Mitani and K.-S. Saito in [11] characterized the strict convexity, uniform convexity and uniform non-squareness of Banach spaces using $\psi$-direct sums $X \bigoplus_{\psi} X$. They showed that, if $t_{0}$ is a unique minimal point, a Banach space $X$ is strictly convex if and only if, for each $x, y \in X$ with $x \neq y$, then

$$
\left\|\left(1-t_{0}\right) x+t_{0} y\right\|<\frac{1}{\psi\left(t_{0}\right)}\left\|\left(\left(1-t_{0}\right) x, t_{0} y\right)\right\|_{\psi}, \quad \psi \in \Psi_{2} .
$$

As for the cases of uniform convexity and uniform non-squareness, they gained some similar results.

Our main purpose of this paper is to give the characterization of $K$ strict convexity, $K$ uniform convexity and uniform non- $l_{1}^{N}$-ness using $\psi$-direct sums $(X \bigoplus X \bigoplus \cdots \bigoplus X)_{\psi}$, we first characterize the $K$ strict convexity using $\psi$ direct sums. We show that, if $\psi$ has a minimal point $s_{0}=\left(t_{1}, t_{2}, \ldots, t_{K}\right)$, and $0<t_{i}<1, i=1,2, \ldots, K$ and $0<\sum_{i=1}^{K} t_{i}<1$, then a Banach space $X$ is $K$ strictly convex if and only if for any $x_{0}, x_{1}, \ldots, x_{K} \in X$, with $x_{0}, x_{1}, \ldots, x_{K}$ linearly independent, we have

$$
\left\|t_{0} x_{0}+t_{1} x_{1}+\cdots+t_{K} x_{K}\right\|<\frac{1}{\psi\left(s_{0}\right)}\left\|\left(t_{0} x_{0}, t_{1} x_{1}, \ldots, t_{K} x_{K}\right)\right\|_{\psi},
$$

where $\sum_{i=0}^{K} t_{i}=1$. As a result, we can give different characterization by choosing different $\psi$. In contrast with the result of K.-I. Mitani and K.-S. Saito [11], the uniqueness of $t_{0}$ is not required, but the linear independence of $x$ and $y$ is necessary. Moreover when $K=1$, we get the characterization of strict convexity. In Section 3, we also characterize the $K$ uniform convexity and 
make Theorem 8 in [11] as our Corollary 3.5. In Section 4, the characterization of uniform non- $l_{1}^{N}$-ness is gained by adding the uniqueness of minimal point.

\section{2. $K$ strict convexity}

A Banach space $X$ is said to be $K$ strictly convex (cf. [14]) if and only if for any $K+1$ elements $x_{0}, x_{1}, \ldots, x_{K}$ in $X$, whenever $\left\|\sum_{i=0}^{K} x_{i}\right\|=\sum_{i=0}^{K}\left\|x_{i}\right\|$, then $x_{0}, x_{1}, \ldots, x_{K}$ are linearly dependent.

The closed unit ball of a Banach space $X$ is $\{x \in X:\|x\| \leq 1\}$ and is denoted by $B_{X}$, the unit sphere of $X$ is $\{x \in X:\|x\|=1\}$ and is denoted by $S_{X}$. It is obvious that when $K=1, X$ is strictly convex.

Proposition 2.1 (cf. [8]). Let $X$ be a Banach space. For all non-zero elements $x_{1}, x_{2}, \ldots, x_{n} \in X$, the following inequality holds:

$$
\begin{aligned}
& \left\|\sum_{j=1}^{n} x_{j}\right\|+\left(n-\left\|\sum_{j=1}^{n} \frac{x_{j}}{\left\|x_{j}\right\|}\right\|\right) \min _{1 \leq j \leq n}\left\|x_{j}\right\| \leq \sum_{j=1}^{n}\left\|x_{j}\right\| \\
\leq & \left\|\sum_{j=1}^{n} x_{j}\right\|+\left(n-\left\|\sum_{j=1}^{n} \frac{x_{j}}{\left\|x_{j}\right\|}\right\|\right) \max _{1 \leq j \leq n}\left\|x_{j}\right\| .
\end{aligned}
$$

Lemma 2.2. Let $X$ be a Banach space. Then the following assertions are equivalent.

(1) $X$ is $K$ strictly convex.

(2) For any $x_{0}, x_{1}, \ldots, x_{K} \in S_{X}$, whenever $\left\|\sum_{i=0}^{K} x_{i}\right\|=K+1$, then $x_{0}, x_{1}, \ldots, x_{K}$ are linearly dependent.

(3) If $x_{0}, x_{1}, \ldots, x_{K} \in S_{X}$ and $x_{0}, x_{1}, \ldots, x_{K}$ are linearly independent, then for any $\left\{t_{i}\right\}_{i=0}^{K}$ satisfying $0<t_{i}<1, \sum_{i=0}^{K} t_{i}=1$, there holds $\left\|\sum_{i=0}^{K} t_{i} x_{i}\right\|<1$.

$\left(3^{\prime}\right)$ If $x_{0}, x_{1}, \ldots, x_{K} \in S_{X}$ and $x_{0}, x_{1}, \ldots, x_{K}$ are linearly independent, then there exists $\left\{t_{i}\right\}_{i=0}^{K}$ with $0<t_{i}<1, \sum_{i=0}^{K} t_{i}=1$, such that $\left\|\sum_{i=0}^{K} t_{i} x_{i}\right\|<1$.

Proof. $(1) \Rightarrow(2)$ is obvious.

$(2) \Rightarrow(1)$ Let any $x_{0}, x_{1}, \ldots, x_{K} \in X \backslash\{0\}$, and $\left\|\sum_{i=0}^{K} x_{i}\right\|=\sum_{i=0}^{K}\left\|x_{i}\right\|$. By Proposition 2.1 we have $\left\|\sum_{i=0}^{K} \frac{x_{i}}{\left\|x_{i}\right\|}\right\|=K+1$. Hence $\frac{x_{0}}{\left\|x_{0}\right\|}, \frac{x_{1}}{\left\|x_{1}\right\|}, \ldots, \frac{x_{K}}{\left\|x_{K}\right\|}$ are linearly dependent, so do $x_{0}, x_{1}, \ldots, x_{K}$.

$(2) \Rightarrow(3)$ Assume that the conclusion falls to hold. Then there exists $\left\{t_{i}\right\}_{i=0}^{K}$ satisfying $0<t_{i}<1, \sum_{i=0}^{K} t_{i}=1$, but $\left\|\sum_{i=0}^{K} t_{i} x_{i}\right\|=1$. Using Proposition 2.1 we have

$$
\begin{aligned}
& \left\|\sum_{i=0}^{K} t_{i} x_{i}\right\|+\left(K+1-\left\|\sum_{i=0}^{K} \frac{x_{i}}{\left\|x_{i}\right\|}\right\|\right) \min _{0 \leq i \leq K}\left\|t_{i} x_{i}\right\| \leq \sum_{i=0}^{K} t_{i}\left\|x_{i}\right\| \\
\leq & \left\|\sum_{i=0}^{K} t_{i} x_{i}\right\|+\left(K+1-\left\|\sum_{i=0}^{K} \frac{x_{i}}{\left\|x_{i}\right\|}\right\|\right) \max _{0 \leq i \leq K}\left\|t_{i} x_{i}\right\| .
\end{aligned}
$$


CHARACTERIZATIONS OF GEOMETRICAL PROPERTIES OF BANACH SPACES 421

Hence $\left\|\sum_{i=0}^{K} \frac{x_{i}}{\left\|x_{i}\right\|}\right\|=K+1$. So $x_{0}, x_{1}, \ldots, x_{K}$ are linearly dependent. Contradiction.

$(3) \Rightarrow(2)$ Clearly.

$(2) \Rightarrow\left(3^{\prime}\right)$ We just need to let $t_{i}=\frac{1}{K+1}, i=0,1, \ldots, K$.

$\left(3^{\prime}\right) \Rightarrow(2)$ If there are $x_{0}, x_{1}, \ldots, x_{K} \in S_{X}$ and satisfying $\left\|\sum_{i=0}^{K} x_{i}\right\|=$ $K+1$, but $x_{0}, x_{1}, \ldots, x_{K}$ are linearly independent. Then there exists $\left\{t_{i}\right\}_{i=0}^{K}$, with $0<t_{i}<1, \sum_{i=0}^{K} t_{i}=1$, and $\left\|\sum_{i=0}^{K} t_{i} x_{i}\right\|<1$. Considering Proposition 2.1 we have

$$
1=\sum_{i=0}^{K} t_{i}\left\|x_{i}\right\| \leq\left\|\sum_{i=0}^{K} t_{i} x_{i}\right\|+\left(K+1-\left\|\sum_{i=0}^{K} x_{i}\right\|\right) \max _{0 \leq i \leq K}\left\|t_{i} x_{i}\right\|,
$$

that is $\left\|\sum_{i=0}^{K} t_{i} x_{i}\right\| \geq 1$, contradiction.

Theorem 2.3. Let $\psi \in \Psi_{K+1}$. Assume that $\psi$ has a minimal point $s_{0}=$ $\left(t_{1}, t_{2}, \ldots, t_{K}\right)$, and $0<t_{i}<1, i=1,2, \ldots, K$ and $0<\sum_{i=1}^{K} t_{i}<1$. Then $a$ Banach space $X$ is $K$ strictly convex if and only if for any $x_{0}, x_{1}, \ldots, x_{K} \in X$, with $x_{0}, x_{1}, \ldots, x_{K}$ linearly independent, we have

$$
\left\|t_{0} x_{0}+t_{1} x_{1}+\cdots+t_{K} x_{K}\right\|<\frac{1}{\psi\left(s_{0}\right)}\left\|\left(t_{0} x_{0}, t_{1} x_{1}, \ldots, t_{K} x_{K}\right)\right\|_{\psi},
$$

where $\sum_{i=0}^{K} t_{i}=1$.

Proof. Assume that $X$ is $K$ strictly convex. Since $\psi(s) \geq \psi\left(s_{0}\right)$ for all $s \in$ $\Delta_{K+1}$, and $t_{0} x_{0}, t_{1} x_{1}, \ldots, t_{K} x_{K}$ are linearly independent, then we have

$$
\begin{aligned}
& \left\|t_{0} x_{0}+t_{1} x_{1}+\cdots+t_{K} x_{K}\right\| \\
< & \left\|t_{0} x_{0}\right\|+\left\|t_{1} x_{1}\right\|+\cdots+\left\|t_{K} x_{K}\right\| \\
= & \left\|\left(t_{0} x_{0}, t_{1} x_{1}, \ldots, t_{K} x_{K}\right)\right\|_{1} \\
\leq & \max _{s \in \Delta_{K+1}} \frac{\psi_{1}(s)}{\psi(s)}\left\|\left(t_{0} x_{0}, t_{1} x_{1}, \ldots, t_{K} x_{K}\right)\right\|_{\psi} \\
= & \frac{1}{\min _{s \in \Delta_{K+1}} \psi(s)}\left\|\left(t_{0} x_{0}, t_{1} x_{1}, \ldots, t_{K} x_{K}\right)\right\|_{\psi} \\
= & \frac{1}{\psi\left(s_{0}\right)}\left\|\left(t_{0} x_{0}, t_{1} x_{1}, \ldots, t_{K} x_{K}\right)\right\|_{\psi} .
\end{aligned}
$$

Conversely for any $x_{i} \in S_{X}, i=0,1, \ldots, K$ with $x_{0}, x_{1}, \ldots, x_{K}$ linearly independent. We have

$$
\begin{aligned}
& \left\|t_{0} x_{0}+t_{1} x_{1}+\cdots+t_{K} x_{K}\right\| \\
< & \frac{1}{\psi\left(s_{0}\right)}\left\|\left(t_{0} x_{0}, t_{1} x_{1}, \ldots, t_{K} x_{K}\right)\right\|_{\psi} \\
= & \frac{1}{\psi\left(s_{0}\right)}\left\|\left(t_{0}, t_{1}, \ldots, t_{K}\right)\right\|_{\psi}
\end{aligned}
$$




$$
=\frac{1}{\psi\left(s_{0}\right)}\left\|\left(1-\sum_{i=1}^{K} t_{i}, t_{1}, \ldots, t_{K}\right)\right\|_{\psi}=1 .
$$

Corollary 2.4. Let $\psi \in \Psi_{2}$. Assume that $\psi$ has a minimal point $t_{0}$. Then a Banach space $X$ is strictly convex if and only if, for each $x, y \in X$ with $x, y$ linearly independent we have

$$
\left\|\left(1-t_{0}\right) x+t_{0} y\right\|<\frac{1}{\psi\left(t_{0}\right)}\left\|\left(\left(1-t_{0}\right) x, t_{0} y\right)\right\|_{\psi} .
$$

Corollary 2.5. If $\psi=\psi_{p} \in \Psi_{K+1}$, when $1<p<\infty, \psi_{p}\left(t_{1}, t_{2}, \ldots, t_{K}\right)=((1-$ $\left.\left.\sum_{i=1}^{K} t_{i}\right)^{p}+t_{1}^{p}+\cdots+t_{K}^{p}\right)^{\frac{1}{p}}$. Note that for any $s \neq\left(\frac{1}{K+1}, \ldots, \frac{1}{K+1}\right), s \in \Delta_{K+1}$, $\psi_{p}(s)>\psi_{p}\left(\frac{1}{K+1}, \ldots, \frac{1}{K+1}\right)=(K+1)^{\frac{1}{p}-1}$. Then a Banach space $X$ is $K$ strictly convex if and only if for any $x_{0}, x_{1}, \ldots, x_{K} \in X$ with $x_{0}, x_{1}, \ldots, x_{K}$ linearly independent, we have

$$
\left\|\frac{x_{0}+x_{1}+\cdots+x_{K}}{K+1}\right\|^{p}<\frac{\left\|x_{0}\right\|^{p}+\cdots+\left\|x_{K}\right\|^{p}}{K+1} .
$$

Theorem 2.3 does not require that $\psi$ is strictly convex. This should be contrasted with the result of [6], i.e., $\left(X_{1} \oplus X_{2} \oplus \cdots \bigoplus X_{n}\right)_{\psi}$ is strictly convex if and only if $X_{1}, X_{2}, \ldots, X_{n}$ are strictly convex respectively and $\psi$ is a strictly convex function on $\Delta_{n}$. Thus, let $\|\cdot\|=\max \left\{\|\cdot\|_{2}, \lambda\|\cdot\|_{1}\right\}\left(\frac{1}{\sqrt{K+1}}<\lambda<1\right)$. Let $\psi$ be the corresponding convex function of $\|\cdot\|$. Then for any $s=\left(s_{1}, \ldots, s_{K}\right) \in$ $\Delta_{K+1}$

$$
\begin{aligned}
& \psi(s)=\left\|\left(1-\sum_{i=1}^{K} s_{i}, s_{1}, \ldots, s_{K}\right)\right\| \\
= & \max \left\{\left\|\left(1-\sum_{i=1}^{K} s_{i}, s_{1}, \ldots, s_{K}\right)\right\|_{2}, \lambda\left\|\left(1-\sum_{i=1}^{K} s_{i}, s_{1}, \ldots, s_{K}\right)\right\|_{1}\right\} \\
= & \max \left\{\psi_{2}(s), \lambda\right\} .
\end{aligned}
$$

Since $\min _{s \in \Delta_{K+1}} \psi_{2}(s)=\frac{1}{\sqrt{K+1}}$. Then

$$
\psi(s)=\left\{\begin{array}{l}
\lambda, \frac{1}{\sqrt{K+1}} \leq \psi_{2}(s) \leq \lambda \\
\psi_{2}(s), \lambda<\psi_{2}(s) \leq 1
\end{array}\right.
$$

For $\psi_{2}(s)$ is continuous on $\Delta_{K+1}$, we have $\min _{s \in \Delta_{K+1}} \psi(s)=\lambda$ and $\psi$ is not strictly convex on $\Delta_{K+1}$. Applying Theorem 2.3, we can give the following characterization using $\psi$ above.

Corollary 2.6. Let $\frac{1}{\sqrt{K+1}}<\lambda \leq 1$. Then a Banach space $X$ is $K$ strictly convex if and only if for any $x_{0}, x_{1}, \ldots, x_{K} \in X$, with $x_{0}, x_{1}, \ldots, x_{K}$ linearly independent, we have

$$
\left\|\frac{x_{0}+x_{1}+\cdots+x_{K}}{K+1}\right\|
$$


CHARACTERIZATIONS OF GEOMETRICAL PROPERTIES OF BANACH SPACES 423

$$
\begin{aligned}
& <\frac{1}{\lambda} \max \left\{\frac{\left(\left\|x_{0}\right\|^{2}+\cdots+\left\|x_{K}\right\|^{2}\right)^{\frac{1}{2}}}{K+1}, \lambda \frac{\left\|x_{0}\right\|+\cdots+\left\|x_{K}\right\|}{K+1}\right\} \\
& =\max \left\{\frac{\left(\left\|x_{0}\right\|^{2}+\cdots+\left\|x_{K}\right\|^{2}\right)^{\frac{1}{2}}}{\lambda(K+1)}, \frac{\left\|x_{0}\right\|+\cdots+\left\|x_{K}\right\|}{K+1}\right\} .
\end{aligned}
$$

\section{3. $K$ uniform convexity}

We say that a Banach space $X$ is $K$ uniformly convex (or $K$ uniformly rotund see [15]) if for any $\varepsilon>0$, there exists some $\delta=\delta(\varepsilon)>0$, such that whenever $x_{0}, x_{1}, \ldots, x_{K} \in S_{X}$ and $\left\|x_{0}+x_{1}+\cdots+x_{K}\right\|>(K+1)-\delta$, we have

$$
\begin{aligned}
& A\left(x_{0}, x_{1}, \ldots, x_{K}\right) \\
\equiv & \sup \left\{\left|\begin{array}{cccc}
1 & 1 & \cdots & 1 \\
f_{1}\left(x_{0}\right) & f_{1}\left(x_{1}\right) & \cdots & f_{1}\left(x_{K}\right) \\
\cdots & \cdots & \cdots & \cdots \\
f_{K}\left(x_{0}\right) & f_{K}\left(x_{1}\right) & \cdots & f_{K}\left(x_{K}\right)
\end{array}\right|,\left\{f_{i}\right\}_{i=1}^{K} \subset B_{X^{*}}\right\}<\varepsilon .
\end{aligned}
$$

In the case of $K=1, X$ is uniformly convex.

Proposition 3.1 (cf. [17]). Let $X$ be a Banach space. Then $X$ is $K$ uniformly convex if and only if for any $K+1$ sequences $\left\{x_{0}^{n}\right\},\left\{x_{1}^{n}\right\}, \ldots,\left\{x_{K}^{n}\right\}$ in $X$, if $\left\|x_{i}^{n}\right\| \rightarrow a, n \rightarrow \infty, i=0,1,2, \ldots, K$ and $\left\|x_{0}^{n}+x_{1}^{n}+\cdots+x_{K}^{n}\right\| \rightarrow(K+1) a$, then

$$
\lim _{n \rightarrow \infty} A\left(x_{0}^{n}, x_{1}^{n}, \ldots, x_{K}^{n}\right)=0 .
$$

Proposition 3.2 (cf. [9]). Let $\left\{x_{1}^{k}\right\}_{k},\left\{x_{2}^{k}\right\}_{k}, \ldots,\left\{x_{n}^{k}\right\}_{k}$ be $n$ sequences in a Banach space $X$ for which the sequences of their norms are convergent. Then the following are equivalent.

(1) $\lim _{k \rightarrow \infty}\left\|\sum_{j=1}^{n} x_{j}^{k}\right\|=\lim _{k \rightarrow \infty} \sum_{j=1}^{n}\left\|x_{j}^{k}\right\|$.

(2) $\lim _{k \rightarrow \infty}\left\|\alpha x_{1}^{k}+\sum_{j=2}^{n} x_{j}^{k}\right\|=\lim _{k \rightarrow \infty}\left(\alpha\left\|x_{1}^{k}\right\|+\sum_{j=2}^{n}\left\|x_{j}^{k}\right\|\right)$ for all $\alpha>0$.

(3) $\lim _{k \rightarrow \infty}\left\|\alpha x_{1}^{k}+\sum_{j=2}^{n} x_{j}^{k}\right\|=\lim _{k \rightarrow \infty}\left(\alpha\left\|x_{1}^{k}\right\|+\sum_{j=2}^{n}\left\|x_{j}^{k}\right\|\right)$ for some $\alpha>0$.

Proposition 3.3 (cf. [13]). Let $\psi \in \Psi_{n}$ and let $x=\left(x_{1}, x_{2}, \ldots, x_{n}\right), y=$ $\left(y_{1}, y_{2}, \ldots, y_{n}\right) \in \mathbb{C}^{n}$. Then

(1) If $|x| \leq|y|$, then $\|x\|_{\psi} \leq\|y\|_{\psi}$.

(2) If $\psi$ is strictly convex and $|x|<|y|$, then $\|x\|_{\psi}<\|y\|_{\psi}$.

For $x=\left(x_{1}, x_{2}, \ldots, x_{n}\right) \in \mathbb{C}^{n}$, denote $|x|$ by $|x|=\left(\left|x_{1}\right|,\left|x_{2}\right|, \ldots,\left|x_{n}\right|\right)$. We say that $|x| \leq|y|$ if $\left|x_{j}\right| \leq\left|y_{j}\right|$ for $1 \leq j \leq n$. Further, we say that $|x|<|y|$ if $|x| \leq|y|$ and $\left|x_{j}\right|<\left|y_{j}\right|$ for some $j$.

Theorem 3.4. Let $\psi \in \Psi_{K+1}$. Assume that $\psi$ has a unique minimal point $s_{0}=\left(t_{1}, t_{2}, \ldots, t_{K}\right)$, with $0<t_{i}<1, \sum_{i=1}^{K} t_{i}<1$. Then a Banach space $X$ is $K$ uniformly convex if and only if for any $\varepsilon>0$, there exists some $\delta>0$, such 
that for any $x_{0}, x_{1}, \ldots, x_{K} \in B_{X}$, satisfying

$$
\left\|t_{0} x_{0}+t_{1} x_{1}+\cdots+t_{K} x_{K}\right\|>(1-\delta) \frac{1}{\psi\left(s_{0}\right)}\left\|\left(t_{0} x_{0}, t_{1} x_{1}, \ldots, t_{K} x_{K}\right)\right\|_{\psi},
$$

where $\sum_{i=0}^{K} t_{i}=1$, then we have $A\left(x_{0}, x_{1}, \ldots, x_{K}\right)<\varepsilon$.

Proof. Let $X$ be a $K$ uniformly convex Banach space. Assume that there exists $\varepsilon_{0}>0$, for any $n \in \mathbb{N}$, there are sequences $\left\{x_{0}^{n}\right\},\left\{x_{1}^{n}\right\}, \ldots,\left\{x_{K}^{n}\right\}$ in $B_{X}$ satisfying

(3) $\left\|t_{0} x_{0}^{n}+t_{1} x_{1}^{n}+\cdots+t_{K} x_{K}^{n}\right\|>\left(1-\frac{1}{n}\right) \frac{1}{\psi\left(s_{0}\right)}\left\|\left(t_{0} x_{0}^{n}, t_{1} x_{1}^{n}, \ldots, t_{K} x_{K}^{n}\right)\right\|_{\psi}$.

But $A\left(x_{0}^{n}, x_{1}^{n}, \ldots, x_{K}^{n}\right) \geq \varepsilon_{0}$.

Since $\left\{\left\|x_{i}^{n}\right\|\right\}_{n=1}^{\infty}, i=0,1, \ldots, K$ and $\left\{\left\|\sum_{i=0}^{K} t_{i} x_{i}^{n}\right\|\right\}_{n=1}^{\infty}$ are bounded sequences, without loss of generality we can let $\left\|x_{i}^{n}\right\| \rightarrow a_{i}(n \rightarrow \infty), i=$ $0,1, \ldots, K$ and $\left\|t_{0} x_{0}^{n}+t_{1} x_{1}^{n}+\cdots+t_{K} x_{K}^{n}\right\| \rightarrow b(n \rightarrow \infty)$. Moreover, we can choose $\left\{\left\|x_{i}^{n}\right\|\right\}_{n=1}^{\infty}$ such that $\max \left\{\left\|x_{i}^{n}\right\|, 0 \leq i \leq K\right\}=1$. Thus $\max \left\{a_{i}, 0 \leq\right.$ $i \leq K\}=1$. From this, $\sum_{i=0}^{K} t_{i} a_{i}>0$. It is clear that $0 \leq a_{i} \leq 1,0 \leq b \leq 1$. Considering the equality (2), we have

$$
\begin{aligned}
& \left(1-\frac{1}{n}\right) \frac{1}{\psi\left(s_{0}\right)}\left\|\left(t_{0} x_{0}^{n}, t_{1} x_{1}^{n}, \ldots, t_{K} x_{K}^{n}\right)\right\|_{\psi} \\
= & \left(1-\frac{1}{n}\right) \frac{1}{\psi\left(s_{0}\right)}\left\|\left(t_{0}\left\|x_{0}^{n}\right\|, t_{1}\left\|x_{1}^{n}\right\|, \ldots, t_{K}\left\|x_{K}^{n}\right\|\right)\right\|_{\psi} \\
< & \left\|t_{0} x_{0}^{n}+t_{1} x_{1}^{n}+\cdots+t_{K} x_{K}^{n}\right\| \\
\leq & t_{0}\left\|x_{0}^{n}\right\|+t_{1}\left\|x_{1}^{n}\right\|+\cdots+t_{K}\left\|x_{K}^{n}\right\| .
\end{aligned}
$$

Let $n \rightarrow \infty$. Then $\frac{1}{\psi\left(s_{0}\right)}\left\|\left(t_{0} a_{0}, t_{1} a_{1}, \ldots, t_{K} a_{K}\right)\right\|_{\psi} \leq t_{0} a_{0}+t_{1} a_{1}+\cdots+t_{K} a_{K}$ holds. Hence

$$
\psi\left(\frac{t_{1} a_{1}}{\sum_{i=0}^{K} t_{i} a_{i}}, \ldots, \frac{t_{K} a_{K}}{\sum_{i=0}^{K} t_{i} a_{i}}\right) \leq \psi\left(s_{0}\right)=\psi\left(t_{1}, t_{2}, \ldots, t_{K}\right) .
$$

From the uniqueness of $s_{0}$, we get $a_{0}=a_{1}=\cdots=a_{K}$. Let us denote them as a. Moreover,

$$
\lim _{n \rightarrow \infty}\left\|\sum_{i=0}^{K} t_{i} x_{i}^{n}\right\|=\lim _{n \rightarrow \infty} \sum_{i=0}^{K}\left\|t_{i} x_{i}^{n}\right\| .
$$

Using Proposition 3.2 we get

$$
\lim _{n \rightarrow \infty}\left\|\frac{1}{t_{0}} t_{0} x_{0}^{n}+\sum_{i=1}^{K} t_{i} x_{i}^{n}\right\|=\lim _{n \rightarrow \infty}\left(\left\|x_{0}^{n}\right\|+\sum_{i=1}^{K}\left\|t_{i} x_{i}^{n}\right\|\right) .
$$

Repeat the similar process above for $K+1$ times, we have

$$
\lim _{n \rightarrow \infty}\left\|\sum_{i=0}^{K} x_{i}^{n}\right\|=\lim _{n \rightarrow \infty} \sum_{i=0}^{K}\left\|x_{i}^{n}\right\|=(K+1) a .
$$


Hence there is $\lim _{n \rightarrow \infty} A\left(x_{0}^{n}, x_{1}^{n}, \ldots, x_{K}^{n}\right)=0$. By Proposition 3.1, it is a contradiction.

Conversely, for any $\varepsilon>0$ there exists some $\delta>0$, such that for any $x_{0}, x_{1}, \ldots, x_{K}$ in $S_{X}$ with $A\left(x_{0}, x_{1}, \ldots, x_{K}\right) \geq \varepsilon$, we have

$$
\begin{aligned}
& \left\|t_{0} x_{0}+t_{1} x_{1}+\cdots+t_{K} x_{K}\right\| \\
\leq & (1-\delta) \frac{1}{\psi\left(s_{0}\right)}\left\|\left(t_{0} x_{0}, t_{1} x_{1}, \ldots, t_{K} x_{K}\right)\right\|_{\psi} \\
\leq & (1-\delta) \frac{1}{\psi\left(s_{0}\right)}\left\|\left(t_{0}, t_{1}, \ldots, t_{K}\right)\right\|_{\psi}=1-\delta .
\end{aligned}
$$

By Proposition 2.1 we have

$$
\begin{aligned}
1=\sum_{i=0}^{K} t_{i}\left\|x_{i}\right\| & \leq\left(K+1-\left\|\sum_{i=0}^{K} x_{i}\right\|\right) \max _{0 \leq i \leq K} t_{i}\left\|x_{i}\right\|+\left\|\sum_{i=0}^{K} t_{i} x_{i}\right\| \\
& \leq\left(K+1-\left\|\sum_{i=0}^{K} x_{i}\right\|\right)+1-\delta .
\end{aligned}
$$

Hence $\left\|\sum_{i=0}^{K} x_{i}\right\| \leq(K+1)-\delta$.

Corollary 3.5 (cf. [11]). Let $\psi \in \Psi_{2}$. Assume that $\psi$ has a unique minimal point $t_{0}$. Then a Banach space $X$ is uniformly convex if and only if, for every $\varepsilon>0$, there exists some $\delta>0$ such that $\|x-y\| \geq \varepsilon, x, y \in B_{X}$ implies

$$
\left\|\left(1-t_{0}\right) x+t_{0} y\right\| \leq(1-\delta) \frac{1}{\psi\left(t_{0}\right)}\left\|\left(\left(1-t_{0}\right) x, t_{0} y\right)\right\|_{\psi} .
$$

Corollary 3.6. Let $\psi(s)=\psi_{p}(s)=\left[\left(1-\sum_{i=1}^{K} s_{i}\right)^{p}+s_{1}^{p}+\cdots+s_{K}^{p}\right]^{\frac{1}{p}}, 1<p<\infty$. Then $\psi_{p}(s)$ has a unique minimal point $s_{0}=\left(\frac{1}{K+1}, \frac{1}{K+1}, \ldots, \frac{1}{K+1}\right)$. A Banach space $X$ is $K$ uniformly convex if and only if for every $\varepsilon>0$, there exists some $\delta>0$ such that for any $x_{0}, x_{1}, \ldots, x_{K}$ in $B_{X}$ satisfying

$$
\left\|\frac{x_{0}+x_{1}+\cdots+x_{K}}{K+1}\right\|^{p}>(1-\delta) \frac{\left\|x_{0}\right\|^{p}+\cdots+\left\|x_{K}\right\|^{p}}{K+1}
$$

implies $A\left(x_{0}, x_{1}, \ldots, x_{K}\right)<\varepsilon$.

\section{Uniform non- $l_{1}^{N}$-ness}

A Banach space $X$ is said to be uniformly non- $l_{1}^{N}$ (cf. $[1,10]$ ) provided there exists $\delta(0<\delta<1)$ such that for any $x_{0}, x_{1}, \ldots, x_{N-1}$ in $S_{X}$, there exists an $N$-tuple of signs $\theta=\left(\theta_{j}\right)$ for which

$$
\left\|\sum_{j=0}^{N-1} \theta_{j} x_{j}\right\| \leq N(1-\delta)
$$

In the case of $N=2, X$ is called uniform non-squareness. As is well known, we may take $x_{0}, x_{1}, \ldots, x_{N-1}$ from $B_{X}$ in the definition (see [8]). 
Lemma 4.1. A Banach space $X$ is uniformly non- $l_{1}^{N}$ if and only if there exist some $s=\left(s_{0}, s_{1}, \ldots, s_{N-1}\right)$, with $\sum_{i=0}^{N-1} s_{i}=1,0<s_{i}<1, i=0,1, \ldots, N-1$, and some $\delta(0<\delta<1)$, such that for any $x_{0}, x_{1}, \ldots, x_{N-1}$ in $B_{X}$, there exists an $N$-tuple of signs $\theta=\left(\theta_{j}\right)$, for which $\left\|\sum_{j=0}^{N-1} \theta_{j} s_{j} x_{j}\right\| \leq 1-\delta$.

Proof. Assume that $X$ is uniformly non- $l_{1}^{N}$. Let $s_{i}=\frac{1}{N}, i=0,1, \ldots, N-1$. For any $x_{0}, x_{1}, \ldots, x_{N-1}$ in $S_{X}$, there exists an $N$-tuple of signs $\theta=\left(\theta_{j}\right)$ and $s=\left(s_{0}, \ldots, s_{N-1}\right)$ with $\sum_{i=0}^{N-1} s_{i}=1,\left\|\sum_{j=0}^{N-1} \theta_{j} s_{j} x_{j}\right\| \leq 1-\delta$. Use Proposition 2.1 we have

$$
\begin{aligned}
1 & =\sum_{j=0}^{N-1}\left\|\theta_{j} s_{j} x_{j}\right\| \\
& \leq\left\|\sum_{j=0}^{N-1} \theta_{j} s_{j} x_{j}\right\|+\left(N-\left\|\sum_{j=0}^{N-1} \theta_{j} x_{j}\right\|\right) \max _{0 \leq i \leq N-1}\left\|\theta_{j} s_{j} x_{j}\right\| \\
& \leq 1-\delta+N-\left\|\sum_{j=0}^{N-1} \theta_{j} x_{j}\right\| .
\end{aligned}
$$

Let $\delta^{\prime}=\frac{\delta}{N}$. Then for any $x_{0}, x_{1}, \ldots, x_{N-1}$ in $S_{X}$, there exists an $N$-tuple of signs $\theta=\left(\theta_{j}\right)$, for which $\left\|\sum_{j=0}^{N-1} \theta_{j} x_{j}\right\| \leq N\left(1-\delta^{\prime}\right)$.

Lemma 4.2. Let $X$ be a Banach space. Then $X$ is uniformly non-l $l_{1}^{N}$ if and only if for any $N$ sequences $\left\{x_{0}^{n}\right\}, \ldots,\left\{x_{N-1}^{n}\right\}$ in $X$ and $\left\|x_{j}^{n}\right\| \rightarrow a(a>0), n \rightarrow$ $\infty, j=0,1, \ldots, N-1,\left\|\sum_{j=0}^{N-1} \theta_{j} x_{j}^{n}\right\| \rightarrow A_{\theta}$ for any $\theta=\left(\theta_{j}\right)$, then there exists an $N$-tuple of signs $\theta=\left(\theta_{j}\right)$ for which

$$
\lim _{n \rightarrow \infty}\left\|\sum_{j=0}^{N-1} \theta_{j} x_{j}^{n}\right\|<N a .
$$

Proof. It is equivalent to prove that: $X$ is not uniformly non- $l_{1}^{N}$ if and only if there exist $N$ sequences $\left\{x_{0}^{n}\right\}, \ldots,\left\{x_{N-1}^{n}\right\}$ in $X$ and $\left\|x_{j}^{n}\right\| \rightarrow a(a>0), n \rightarrow$ $\infty, j=0,1, \ldots, N-1$, for any $N$-tuple of signs $\theta=\left(\theta_{j}\right)$ there holds

$$
\lim _{n \rightarrow \infty}\left\|\sum_{j=0}^{N-1} \theta_{j} x_{j}^{n}\right\|=N a .
$$

Without loss of generality, let $a=1$. On one hand, since $\left\|x_{j}^{n}\right\| \rightarrow 1, j=$ $0,1, \ldots, N-1$, we can assume that $\left\|x_{j}^{n}\right\|>0$, then $\left\{\frac{x_{j}^{n}}{\left\|x_{j}^{n}\right\|}\right\} \subseteq S_{X}$. In addition, we have

$$
\left|\left\|\sum_{j=0}^{N-1} \theta_{j} \frac{x_{j}^{n}}{\left\|x_{j}^{n}\right\|}\right\|-\left\|\sum_{j=0}^{N-1} \theta_{j} x_{j}^{n}\right\|\right|
$$


CHARACTERIZATIONS OF GEOMETRICAL PROPERTIES OF BANACH SPACES 427

$$
\begin{aligned}
& \leq\left\|\sum_{j=0}^{N-1} \theta_{j}\left(\frac{x_{j}^{n}}{\left\|x_{j}^{n}\right\|}-x_{j}^{n}\right)\right\| \leq \sum_{j=0}^{N-1}\left\|\frac{x_{j}^{n}}{\left\|x_{j}^{n}\right\|}-x_{j}^{n}\right\| \\
& =\sum_{j=0}^{N-1}\left|\frac{1}{\left\|x_{j}^{n}\right\|}-1\right| \cdot\left\|x_{j}^{n}\right\| \rightarrow 0(n \rightarrow \infty) .
\end{aligned}
$$

Hence

$$
\lim _{n \rightarrow \infty}\left\|\sum_{j=0}^{N-1} \theta_{j} \frac{x_{j}^{n}}{\left\|x_{j}^{n}\right\|}\right\|=\lim _{n \rightarrow \infty}\left\|\sum_{j=0}^{N-1} \theta_{j} x_{j}^{n}\right\|=N .
$$

By definition $X$ is not uniformly non- $l_{1}^{N}$.

The converse is obvious from the definition of uniform non- $l_{1}^{N}$-ness.

Theorem 4.3. Let $\psi \in \Psi_{N}$. Assume that $\psi$ has a unique minimal point $s=\left(s_{1}, s_{2}, \ldots, s_{N-1}\right)$ with $\sum_{i=1}^{N-1} s_{i}<1,0<s_{i}<1, i=1,2, \ldots, N-1$. Then a Banach space $X$ is uniformly non- $l_{1}^{N}$ if and only if there exists $\delta(0<\delta<1)$ such that for any $x_{0}, x_{1}, \ldots, x_{N-1}$ in $B_{X}$, there exists an $N$-tuple of signs $\theta=\left(\theta_{j}\right)$, for which

$$
\left\|\sum_{j=0}^{N-1} s_{j} \theta_{j} x_{j}\right\| \leq(1-\delta) \frac{1}{\psi(s)}\left\|\left(s_{0} x_{0}, s_{1} x_{1}, \ldots, s_{N-1} x_{N-1}\right)\right\|_{\psi},
$$

where $s_{0}=1-\sum_{i=1}^{N-1} s_{j}$.

Proof. Let $X$ be a uniformly non- $l_{1}^{N}$ Banach space. Assume that the conclusion fails to hold. Then for $\delta_{n}=\frac{1}{n}, n \in \mathbb{N}$, there exist sequences $\left\{x_{j}^{n}\right\}$ in $B_{X}$, $j=0,1, \ldots, N-1$, for any $N$-tuple of signs $\theta=\left(\theta_{j}\right)$, we have

$$
\begin{aligned}
& \left\|\sum_{j=0}^{N-1} s_{j} \theta_{j} x_{j}^{n}\right\| \\
> & \left(1-\frac{1}{n}\right) \frac{1}{\psi(s)}\left\|\left(s_{0} x_{0}^{n}, s_{1} x_{1}^{n}, \ldots, s_{N-1} x_{N-1}^{n}\right)\right\|_{\psi} \\
= & \left(1-\frac{1}{n}\right) \frac{1}{\psi(s)}\left\|\left(s_{0}\left\|x_{0}^{n}\right\|, s_{1}\left\|x_{1}^{n}\right\|, \ldots, s_{N-1}\left\|x_{N-1}^{n}\right\|\right)\right\|_{\psi} .
\end{aligned}
$$

Because $\left\{\left\|x_{j}^{n}\right\|\right\}_{n=1}^{\infty}, j=0,1, \ldots, N-1$ are bounded sequences, we just let $\left\|x_{j}^{n}\right\| \rightarrow a_{j}(n \rightarrow \infty), j=0,1, \ldots, N-1$. Without loss of generality, we can choose $\left\{\left\|x_{j}^{n}\right\|\right\}_{n=1}^{\infty}$ such that $\max \left\{\left\|x_{j}^{n}\right\|, 0 \leq j \leq N-1\right\}=1$. As $a_{j}$ is the limit of $\left\{\left\|x_{j}^{n}\right\|\right\}_{n=1}^{\infty}$, we get $\max \left\{a_{j}, 0 \leq j \leq N-1\right\}=1$. Thus $\sum_{j=0}^{N-1} s_{j} a_{j}>0$. In (4) let $n \rightarrow \infty$, then there is

$$
\frac{1}{\psi(s)}\left\|\left(s_{0} a_{0}, s_{1} a_{1}, \ldots, s_{N-1} a_{N-1}\right)\right\|_{\psi} \leq \sum_{j=0}^{N-1} s_{j} a_{j} .
$$


From this we get

$$
\psi\left(\frac{s_{1} a_{1}}{\sum_{j=0}^{N-1} s_{j} a_{j}}, \ldots, \frac{s_{N-1} a_{N-1}}{\sum_{j=0}^{N-1} s_{j} a_{j}}\right) \leq \psi\left(s_{1}, \ldots, s_{N-1}\right) .
$$

By the uniqueness of $s=\left(s_{1}, s_{2}, \ldots, s_{N-1}\right)$, we get $a_{0}=a_{1}=\cdots=a_{N-1}$, denote them as $a$. In addition, from (4) we get $\lim _{n \rightarrow \infty}\left\|\sum_{j=0}^{N-1} s_{j} \theta_{j} x_{j}^{n}\right\|=1=$ $\lim _{n \rightarrow \infty} \sum_{j=0}^{N-1}\left\|s_{j} \theta_{j} x_{j}^{n}\right\|$. Using Proposition 3.2 there holds

$$
\lim _{n \rightarrow \infty}\left\|\sum_{j=0}^{N-1} \theta_{j} x_{j}^{n}\right\|=\lim _{n \rightarrow \infty} \sum_{j=0}^{N-1}\left\|\theta_{j} x_{j}^{n}\right\|=N a .
$$

It's a contradiction by Lemma 4.2 .

On the other hand, for any $x_{0}, x_{1}, \ldots, x_{N-1}$ in $B_{X}$

$$
\begin{aligned}
\left\|\sum_{j=0}^{N-1} s_{j} \theta_{j} x_{j}\right\| & \leq(1-\delta) \frac{1}{\psi(s)}\left\|\left(s_{0} x_{0}, s_{1} x_{1}, \ldots, s_{N-1} x_{N-1}\right)\right\|_{\psi} \\
& \leq(1-\delta) \frac{1}{\psi(s)}\left\|\left(s_{0}, s_{1}, \ldots, s_{N-1}\right)\right\|_{\psi} \\
& =1-\delta .
\end{aligned}
$$

We claim that $X$ is uniformly non- $l_{1}^{N}$ by Lemma 4.1 .

Corollary 4.4. Let $\psi \in \Psi_{2}$. Assume that $\psi$ has the unique minimum at $t=t_{0}\left(0<t_{0}<1\right)$. Then a Banach space $X$ is uniformly non-square if and only if there exists some $\delta(0<\delta<1)$ such that for any $x, y \in B_{X}$ implies

$$
\min \left\{\left\|\left(1-t_{0}\right) x+t_{0} y\right\|,\left\|\left(1-t_{0}\right) x-t_{0} y\right\|\right\} \leq(1-\delta) \frac{1}{\psi\left(t_{0}\right)}\left\|\left(\left(1-t_{0}\right) x, t_{0} y\right)\right\|_{\psi} \text {. }
$$

Corollary 4.5. A Banach space $X$ is uniformly non- $l_{1}^{N}$ if and only if there exists some $\delta(0<\delta<1)$ such that for any $x_{0}, x_{1}, \ldots, x_{N-1}$ in $B_{X}$, there exists an $N$-tuple of signs $\theta=\left(\theta_{j}\right)$ for which

$$
\left\|\frac{\sum_{j=0}^{N-1} \theta_{j} x_{j}}{N}\right\|^{p} \leq(1-\delta) \frac{\left\|x_{0}\right\|^{p}+\cdots+\left\|x_{N-1}\right\|^{p}}{N},
$$

where $1<p<\infty$.

Proof. We only need to let $\psi(t)=\psi_{p}(t)=\left[\left(1-\sum_{i=1}^{N-1} t_{i}\right)^{p}+t_{1}^{p}+\cdots+t_{N-1}^{p}\right]^{\frac{1}{p}}$ in Theorem 4.3.

Acknowledgment. The authors thank the anonymous referees for the constructive comments and suggestions. Especially, thanks for bringing forward Keni-Ichi Mitani and Kichi-Suke Saito's research results. 


\section{References}

[1] B. Beauzamy, Introduction to Banach Spaces and Their Geometry, 2nd ed., North Holland, 1985.

[2] F. F. Bonsall and J. Duncan, Numerical Ranges. II, London Mathematical Society Lecture Notes Series, No. 10. Cambridge University Press, New York-London, 1973.

[3] S. Dhompongsa, A. Kaewcharoen, and A. Kaewkhao, Fixed point property of direct sums, Nonlinear Anal. 63 (2005), 2177-2188.

[4] S. Dhompongsa, A. Kaewkhao, and S. Saejung, Uniform smoothness and U-convexity of $\psi$-direct sums, J. Nonlinear Convex Anal. 6 (2005), no. 2, 327-338.

[5] P. N. Dowling and B. Turett, Complex strict convexity of absolute norms on $\mathbb{C}^{n}$ and direct sums of Banach spaces, J. Math. Anal. Appl. 323 (2006), no. 2, 930-937.

[6] M. Kato, K.-S. Saito, and T. Tamura, On $\psi$-direct sums of Banach spaces and convexity, J. Aust. Math. Soc. 75 (2003), no. 3, 413-422.

[7] Uniform non-squareness of $\psi$-direct sums of Banach spaces $X \bigoplus_{\psi} Y$, Math. Inequal. Appl. 7 (2004), no. 3, 429-437.

[8] - Sharp triangle inequality and its reverse in Banach spaces, Math. Inequal. Appl. 10 (2007), no. 2, 451-460.

[9] _ Uniform non-l ${ }_{1}^{n}$-ness of $\psi$-direct sums of Banach spaces, J. Nonlinear Convex Anal. 11 (2010), no. 1, 13-33.

[10] R. E. Megginson, An Introduction to Banach Spaces Theory, Springer, 1998.

[11] K.-I. Mitani and K.-S. Saito, A note on geometrical properties of Banach spaces using $\psi$-direct sums, J. Math. Anal. Appl. 327 (2007), no. 2, 898-907.

[12] K.-S. Saito and M. Kato, Uniform convexity of $\psi$-direct sums of Banach spaces, J. Math. Anal. Appl. 277 (2003), no. 1, 1-11.

[13] K.-S. Saito, M. Kato, and Y. Takahashi, Absolute norms on $\mathbb{C}^{n}$, J. Math. Anal. Appl. 252 (2000), no. 2, 879-905.

[14] I. Singer, On the set of best approximation of an element in a normed linear space, Rev. Math. Pures Appl. 5 (1960), 383-402.

[15] F. Sullivan, A generalization of uniformly rotund Banach spaces, Can. J. Math. 31 (1979), no. 3, 628-636.

[16] Y. Takahashi, M. Kato, and K.-S. Saito, Strict convexity of absolute norms on $\mathbb{C}^{2}$ and direct sums of Banach spaces, J. Inequal. Appl. 7 (2002), no. 2, 179-186.

[17] X. T. Yu, E. B. Zang, and Z. Liu, On KUR Banach spaces, J. East China Normal Univ. Nature Science Edition. 1 (1981), 1-8.

ZHIHUA ZHANG

School of Mathematical Sciences

University of Electronic Science And Technology of China

Chengdu 611731, Sichuan Province, P. R. China

E-mail address: zhihuamath@yahoo.cn

LAN SHU

School of Mathematical Sciences

University of Electronic Science and Technology of China

Chengdu 611731, Sichuan Province, P. R. China

E-mail address: shul@uestc.edu.cn

JUN ZHENG

School of Mathematics and Statistics

LANZHOU UNIVERSITY

Lanzhou 730000, Gansu Province, P. R. China

E-mail address: zhengj_2010@lzu.edu.cn 
YULING YANG

School of Mathematical Sciences

University of Electronic Science and Technology of China

Chengdu 611731, Sichuan Province, P. R. China

E-mail address: yulingkathy@sina.com 